\title{
Proporcionaldade da carga DURANTE AULAS DE NATAÇÃO DE ADULTOS
}

\author{
Yvetta Macejková1 macejkova@fsport.uniba.sk \\ Dana Masaryková ${ }^{1}$ masarykova@ fsport.uniba.sk \\ Jela Labudová ${ }^{1}$ labudova@ fsport.uniba.sk
}

doi:10.3900/fpj.7.3.180.p

Macejková Y, Masaryková D, Labudová J. Proporcionalidade da carga durante aulas de natação de adultos. Fit Perf J. 2008 maijun; $7(3): 180-4$.

\section{RESUMO}

Introdução: Atividades físicas no ambiente aquático são essenciais para a saúde do homem. 0 nível de habilidades aquáticas é condicionado pelo nível da técnica natatória. A melhoria da técnica não é condicionada pelo tempo e constitui uma parte muito importante das aulas de natação. Este trabalho visa trazer conhecimento sobre a diferenciada eficiência da carga física relacionada à melhoria das técnicas natatórias em adultos. 0 objetivo deste estudo foi 0 de encontrar a intensidade da carga durante aulas de natação para adultos. Q ueremos adaptar o conteúdo das aulas para melhorar as técnicas natatórias. Materiais e Métodos: Monitoramos duas mulheres (34 e 35 anos de idade) e um homem (44 anos) durante um curso de natação para adultos (20 aulas), duas vezes por semana. Todos os adultos monitorados estavam no mesmo nível técnico. Eles conseguiam nadar $50 \mathrm{~m}$ de peito e costas. Usamos a Fórmula de Carvonen para especificar a zona aeróbica. 0 mínimo contabilizado foi com $0,6 \mathrm{FC}_{\text {máx }}($ ou 0,5$)$ e máximo com $0,8 \mathrm{FC}_{\text {máx }}$ (ou 0,7$)$. Resultados: 0 s seguintes fatores influenciaram na proporcionalidade da carga durante as aulas: experiência anterior na água (bloqueio psíquico); predisposição so mática; qualidade das habilidades natatórias; idade. A freqüência cardíaca mais alta foi medida durante as aulas que visavam a melhoria das técnicas de nado crawl e costas. Discussão: As aulas de natação para adultos podem ser alocadas entre as atividades físicas em ambiente aquático que desenvolvem condição física e têm, em sua maioria, caráter aeróbico.

\section{PALAVRAS-CHAVE}

Natação, Educação Física e Treinamento, Atividade Física.

${ }^{1}$ Comenius University - Faculty of Physical Education and Sport - Department of Outdoor Sports and Swimming - Bratislava - Slovakia

Copyright@ 2008 por Colégio Brasileiro de Atividade Física, Saúde e Esporte

Fit Perf J Rio de Janeiro | 7 | 3 | 180-184 | mai/jun 2008 


\section{Proporcionalidad de la carga durante CLASES de nataCión de adultos}

\section{ABSTRACT}

Introduction: Physical activities in water environment are essential for man's health. The level of swimming abilities is conditioned by level of swimming technique. Improving of swimming technique is not time conditioned and therefore it makes a very important part of swimming lessons. 0 ur paper brings knowledge about the differentiated efficiency of on physical load while improving swimming technique of adults. The aim of the paper was to find out intensity of load during adult's swimming lessons. We wanted to adapt the content of the lessons to improve swimming technique. Materials and Methods: We monitored two women (34years and 35years) and one man (44years) during a swimming course for adults (20 lessons), twice a week. All monitored adults were on the same swimming level, they were able to swim $50 \mathrm{~m}$ breaststroke and backstroke. We used the Carvonen Formula for specifying the aerobic zone. Minimum was counted with $0.6 \mathrm{HR}_{\max }$ (or 0.5 ) and maximum with $0.8 \mathrm{HR}_{\max }$ (or 0.7 ). Results: The following factors influenced the proportionality of load during the lessons: previous water experience (psychic blocks), somatic predispositions, quality of swimming skills, age. The highest heart rate was measured during lessons of improving crawl and backstroke technique. Discussion: The swimming lessons of adults can be placed among the physical activities in water environment which develop physical condition and have mostly aerobic character.

\section{KEYWORDS}

Swimming, Physical Education and Training, Actividad Física.

\section{Proporcionalidad de la carga durante clases de natación de adultos \\ RESUMEN}

Introducción: Actividades físicas en el ambiente acuático son esenciales para la salud del hombre. El nivel de habilidades acuáticas es condicionado por el nivel de la técnica natatoria. La mejoría de la técnica no es condicionada por el tiempo y constituye una parte muy importante de las clases de natación. Este trabajo visa traer conocimiento sobre la diferenciada eficiencia de la carga física relacionada a la mejoría de las técnicas natatorias en adultos. El objetivo de este estudio fue lo de encontrar la intensidad de la carga durante clases de natación para adultos. Q ueremos adaptar el contenido de las clases para mejorar las técnicas natatorias. Materiales y Métodos: Vigilamos dos mujeres (34 y 35 años de edad) y un hombre (44 años) durante un curso de natación para adultos (20 clases), dos veces a la semana. Todos los adultos vigilados estaban en el mismo nivel técnico. Ellos conseguían nadar $50 \mathrm{~m}$ de pecho y espalda. Usamos la Fórmula de Carvonen para especificar la zona aeróbica. El mínimo contabilizado fue con $0,6 \mathrm{FC}_{\text {máx }}(0$ 0,5) y máximo con $0,8 \mathrm{FC}_{\text {máx }}(0 \quad 0,7)$. Resultados: Los siguientes factores influenciaron en la proporcionalidad de la carga durante las clases: experiencia anterior en el agua (bloqueo psíquico); predisposición somática; calidad de las habilidades natatorias; edad. La frecuencia cardiaca más alta fue medida durante las clases que visaban la mejoría de las técnicas de nado braza y espalda. Discusión: Las clases de natación para adultos pueden ser destinadas entre las actividades físicas en ambiente acuático que desarrollan condición física y tienen, en su mayor parte, carácter aeróbico.

\section{PALABRAS CLAVE}

Natación, Educación y Entrenamiento Físico, Physical Activity.

\section{INTRODUÇÃO}

N otamos um interesse incomum em atividades físicas de adultos no ambiente aquático, durante os últimos 15 anos. A razão principal para isto encontra-se nas mudanças no estilo de vida de cada indivíduo, no qual o papel mais importante é representado pela saúde. Diferentes grupos da população em idade produtiva estão aumentando o interesse em atividades que apóiam o estilo de vida saudável.

Natação é considerada como uma atividade insubstituível, tendo influência positiva nos sistemas motor e cardiovascular, representando um papel importante nas seguintes tarefas:

- compensação de movimento em terra (posição horizontal, trabalho de grupos musculares agonistas e antagonistas);

- $\quad$ estimulação do organismo através de padrões do ambiente aquático (resistência, flutuabilidade);

- adaptação do organismo no ambiente aquático (mecânico, térmico e influência química); 
- função corretiva (nadar requer a utilização de ambas as metades do corpo e qualquer movimento na água afeta o corpo por completo).

0 ambiente aquático permite fazer uma gama extensiva de atividades físicas, graças à sua quantidade ilimitada de influências mutáveis. No passado, a palavra natação era usada para toda atividade realizada na água (natação infantil, natação para deficientes, natação de reabilitação, etc.) Atualmente, as atividades são ordenadas em três grupos básicos, de acordo com o objetivo da atividade física, sendo classificadas como ${ }^{1}$ :

- atividades com o objetivo de desempenho desportivo (natação como esporte, nado sincronizado, natação de longa distância, etc.);

- atividades que têm caráter estimulante e adaptável (mergulho, aquafitness, natação como fitness, etc.);

- atividades com o objetivo de melhorar, proteger e estabilizar a saúde (natação para deficientes, natação master, natação para gestantes, natação básica, etc.).

A atividade física na água é realizada dentro de certo conteúdo e forma. Por exemplo, um adulto precisa freqüentar 20 aulas de natação com uma assiduidade maior do que duas vezes por semana. $Q$ uando consideramos quem é nadador e quem não é, usamos uma definição básica: nadador é uma pessoa que nada $200 \mathrm{~m}$ seguramente sem se cansar, mergulha em água profunda e nada debaixo da água.

$\mathrm{Na}$ Eslováquia, recentemente foi dada uma maior atenção para atividades aquáticas em função de mudanças demográficas (maior porcentagem de pessoas mais velhas) e do aumento no número de doenças da civilização. 0 uso de atividades aquáticas para a saúde e aptidão é condicionada pelas habilidades. $\mathrm{Na}$ Eslováquia não temos muitas possibilidades de utilizar o ambiente aquático sem a habilidade de nadar. 95\% das piscinas na Eslováquia, que são usadas pela população, são profundas. A natação, como uma atividade física, representa um papel importante na vida de todo homem, graças ao seu caráter especial de locomoção. A prevenção mais importante é salvar a vida.

O Departamento de Natação da Faculdade de Educação Física e Esporte fez uma pesquisa voltada para a eficiência das atividades físicas na água. Elas costumam ter eficiência fisiológica diferente, em termos de intensidade. A intensidade é condicionada pela estrutura das atividades físicas. Uma carga aeróbico-anaeróbica foi constatada na natação esportiva (80\%-90\% da freqüência cardíaca máxima $)^{2}$; em aquafitness e natação fitness encontrou-se a taxa mais alta na zona aeróbica $\left(60 \%-80 \% \mathrm{FC}_{\text {máx }}\right)^{3}$; e na natação básica e na natação voltada para a saúde foi encontrada a porcentagem mais alta na área da saúde $\left(50 \%-60 \% \mathrm{FC}_{\text {máx }}\right)^{4}$. 0 objetivo da pesquisa foi trazer proporções objetivas de intensidade nas diferentes atividades físicas na água. A base da carga física na água vem da simbiose mútua dos princípios da água (flutuabilidade e pressão) e a estrutura dos movimentos natatórios.

0 papel traz resultados sobre a intensidade da carga durante as aulas de natação de adultos. 0 objetivo foi considerar o nível apropriado de intensidade durante estas aulas. Consideramos a eficiência fisiológica das aulas de natação através da freqüência cardíaca.

\section{MATERIAIS E MÉTODOS}

Preparamos um programa de 20 lições para melhorar a técnica natatória da população adulta. 0 curso levou dois meses, com a freqüência de duas vezes por semana. 0 s adultos na idade de 26-64 anos começaram o curso como meio-nadadores e, ao término do curso, $70 \%$ deles nadaram $100 \mathrm{~m}$ ininterruptamente. A intensidade da carga

Figura 1 - Curva fisiológica da carga durante a aula de natação de adultos (L.D.)

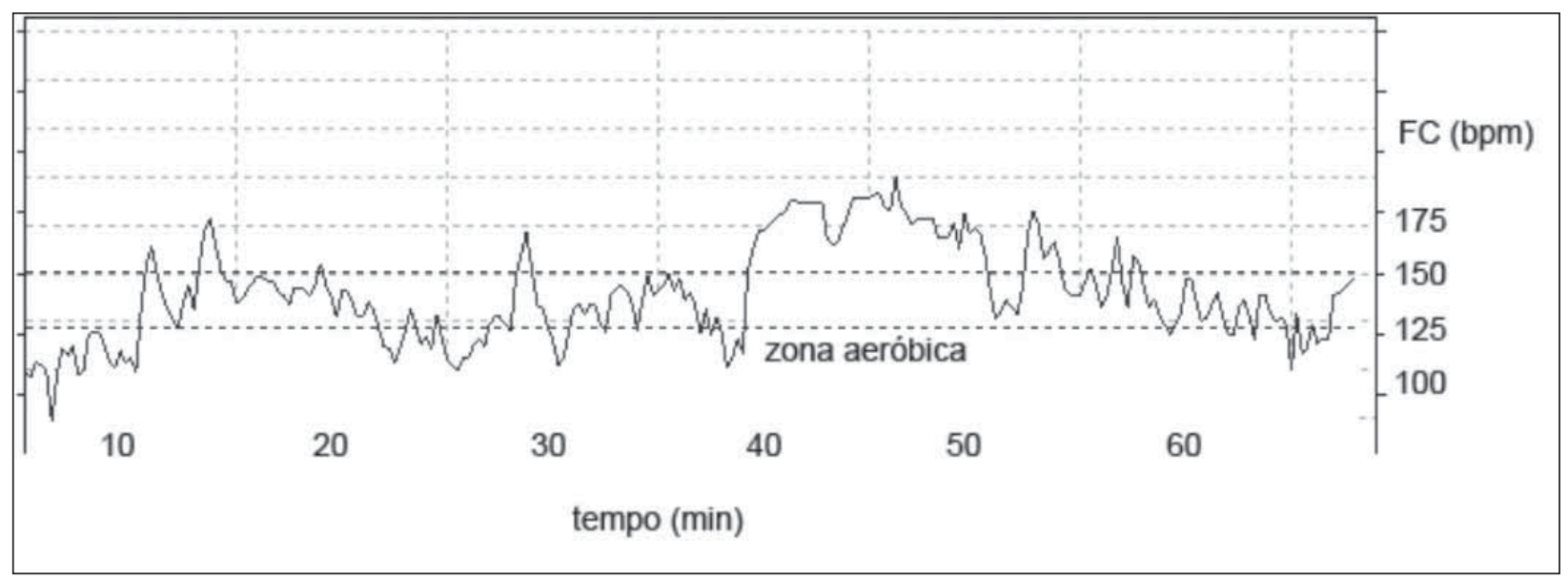


Figura 2 - Comparação das zonas de intensidade com dois modos diferentes de especificar a zona aeróbica (L.D.)

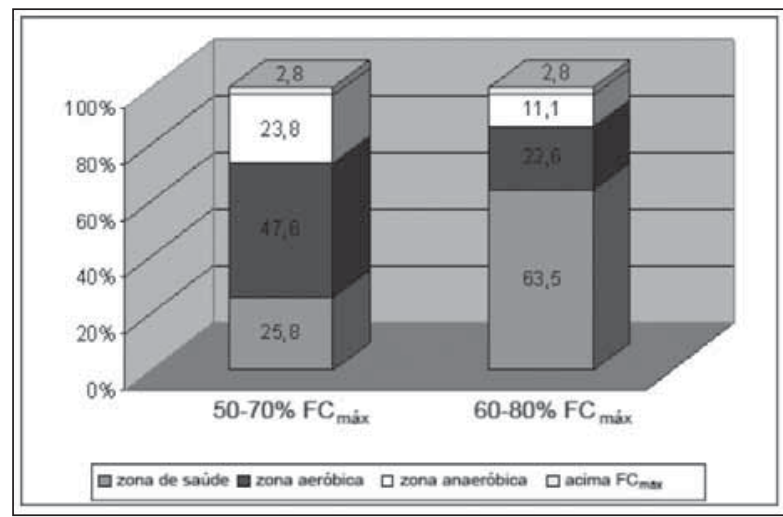

Figura 4 - Proporcionalidade da carga de três adultos diferentes (19a aula)

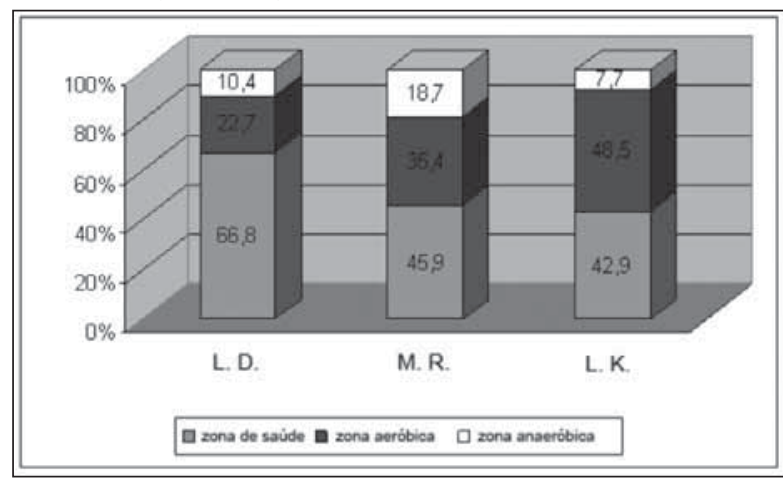

foi monitorada por especialistas. Usamos a Fórmula de Carvonen para especificar a zona aeróbica. 0 mínimo foi contado como $0,6 \mathrm{FC}_{\text {máx }}($ ou 0,5$)$ e o máximo como $0,8 \mathrm{FC}_{\text {máx }}$ (ou 0,7). Supusemos que, para desenvolver a saúde relacionando ao conteúdo das aulas (técnica natatória), seria mais recomendável usar uma porcentagem mais baixa de carga do que é recomendado para melhorar o nível de aptidão.

Mínimo da zona aeróbica:

$$
\text { [(220-idade) } \left.-\mathrm{FC}_{\text {res }}\right] \times 0,5+\mathrm{FC}_{\text {resi }} \text {; }
$$

Máximo da zona aeróbica:

$$
\text { [(220-idade) } \left.-\mathrm{FC}_{\text {res }}\right] \times 0,7+\mathrm{FC}_{\text {res. }}
$$

Monitoramos duas mulheres de 34 anos, L.D. e M.R., e um homem de 44 anos, L.K. Todos eles estavam no mesmo nível técnico.

\section{RESULTADOS}

A curva fisiológica de carga, durante a $2^{a}$ aula de natação de L.D., possui certa dinâmica de acordo com os meios usados. 0 s primeiros 5 min foram de nado livre, seguidos de aquecimento com corridinha em água funda, e então técnicas de crawl e nado de costas. A carga mais alta foi monitorada durante o jogo de pólo aquático e os
Figura 3 - Proporcionalidade de carga de três adultos diferentes ( 8 a aula)

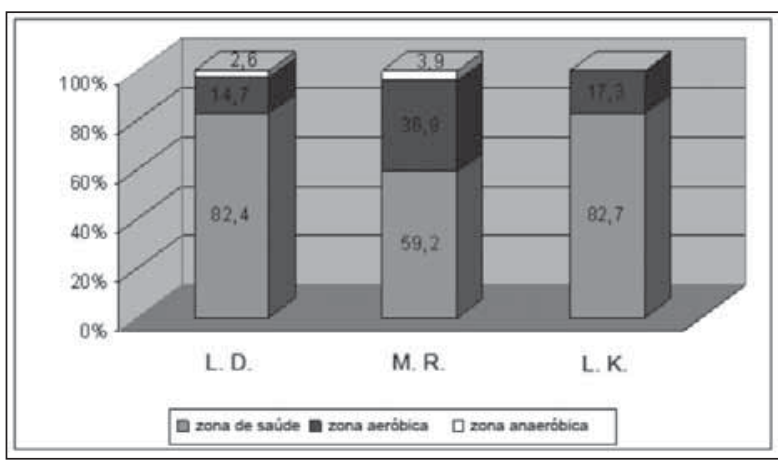

Figura 5 - Intensidade de carga durante várias aulas de natação (L.D.)

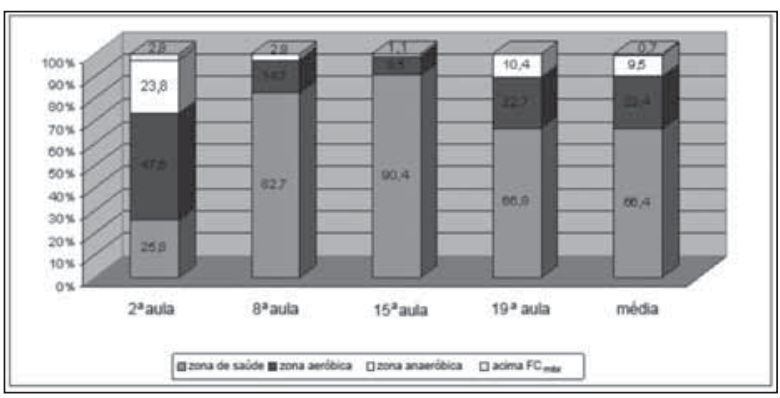

últimos $15 \mathrm{~min}$ foram de nado de peito (Figura1). As linhas horizontais na figura ilustram a zona aeróbica, delimitada por 0,5 e 0,7FC máx

A razão para usar uma zona aeróbica mais baixa é a intenção de melhorar a técnica natatória, o que é necessário em termos de aprendizagem motora. Durante as lições tentamos explicar o movimento certo e os adultos devem perceber se fazem o movimento corretamente ou não. Com exceção a isso, de acordo com muitos fisiologistas, o corpo humano reage à pressão da água com uma taxa cardíaca mais baixa ${ }^{5}$.

As diferenças na porcentagem são apresentadas nas zonas de carga (Figura 2). A mesma aula da L.D. foi dividida por uma mais baixa (50\% a 70\%) e uma mais alta (60\% a 80\%) porcentagem de carga. A natação básica está entre as atividades físicas na água que tem como objetivo prevenir e estabilizar a saúde. E esta é, em parte, a razão porque a maior parte da aula é realizada na zona da saúde (63,5\% em média). Podemos encontrar as seguintes zonas de carga, na literatura:

- $\quad$ 50-60\% $\mathrm{FC}_{\text {máx }}$ - queima de gordura, satisfatório para iniciantes e pessoas mais idosas;

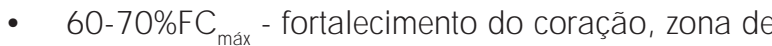
saúde; 
- $\quad 70-80 \% \mathrm{FC}_{\text {máx }}$ - zona aeróbica, aumento da aptidão física.

Um fato importante na avaliação da natação de adultos é que todas as zonas de intensidade apareceram durante as aulas. Suas quantidades e porcentagens de carga são altamente individuais e condicionadas por idade e qualidade das habilidades do nado.

As Figuras 4 e 5 documentam o mesmo conteúdo da aula com reações diferentes dos três adultos. Durante a $8^{a}$ aula, onde a parte principal foi constituída de técnica de crawl, a intensidade mais alta foi monitorada na mulher de 34 anos (M.R.) e durante a 19a aula a intensidade mais alta foi medida no homem mais velho, de 44 anos (L.K.). 0 conteúdo da 19a aula teve como foco a técnica das três braçadas de natação e a qualidade de administrar cada técnica particular foi mostrada claramente. A mulher com mais baixa taxa de carga física (L.D.) mostrou melhor desempenho na técnica natatória.

Q uando avaliamos os mesmos meios para melhorar a técnica, encontramos grandes diferenças nos sentimentos subjetivos e na carga real, monitoradas por especialistas. $0 \mathrm{~s}$ meios usados eram muito freqüentemente descritos como difíceis, mas a taxa cardíaca não confirmou isso. $Q$ uando um treinador ou um professor prepara o conteúdo das aulas, ele deve escolher cuidadosamente os meios de treinamento em termos de dificuldade, volume e intensidade. As habilidades, assim como os elementos particulares da técnica natatória, requerem certo tempo para melhorar, sob a supervisão de um treinador. $\mathrm{Nem}$ todo mundo aprende a mesma braçada facilmente. N ossos resultados mostraram um maior interesse das mulheres no nado de peito, mas havia mulheres que aprenderam nado de costas e crawl mais rapidamente (quase $1 / 3$ de todos os participantes que fizeram parte no curso nos últimos 5 anos). Colocamos nas aulas exercícios especiais e elementos (exercícios com diferentes equipamentos e em várias profundidades, jogos de água) para aumentar a precisão na água. 0 s jogos mais populares eram: pólo aquático em água rasa; mergulho em águas profundas; e jogos de relevo, nos quais as dificuldades do nado foram modificadas.

A intensidade de carga da mesma mulher (L.D.), durante quatro aulas de natação, é apresentada na Figura 5. A taxa de porcentagem da zona aeróbica e de saúde é altamente diferenciada e é condicionada pela dificuldade de meios e a duração do exercício. A intensidade mais baixa foi monitorada durante as aulas de aprendizagem, onde os elementos novos eram aprendidos com intervalos bastante longos de descanso. A última coluna, na Figura 5, apresenta a carga média de todas as aulas de natação, mostrando a proporcionalidade de carga física durante as aulas de natação de adultos. Portanto, podemos colocar esta atividade física entre as atividades com caráter saúde-aeróbico.

\section{DISCUSSÃO}

Com base nos nossos resultados das aulas de natação de adultos, sugerimos avaliar também a intensidade em zonas mais baixas. $Q$ uando realizamos atividades físicas com intenções específicas (por exemplo: natação sênior, melhoria da técnica ou natação para gestantes), é necessário prestar atenção às zonas de intensidade mais baixas, porque o nível de aptidão da população comum é, geralmente, baixo.

A avaliação da singularidade do processo pedagógico em reais condições é um modo eficaz para fazer com que o processo se torne mais eficiente. A intensidade da atividade física é um indicador importante de qualidade e também permite ótimas mudanças do conteúdo das aulas. A intensidade de partes particulares das aulas é condicionada pela escolha de exercício real em termos de demandas técnicas, duração do exercício e sua intensidade. Exercícios que são mais difíceis em termos de freqüência - estilo livre com pernada, nado de costas com pernada - também têm expectativas maiores em termos de carga.

A proporcionalidade da intensidade durante as aulas é influenciada pela idade, qualidade das habilidades do nado, estrutura do corpo e duração da preparação. As aulas de natação básica podem ser colocadas entre os exercícios na zona saúde-aeróbica (50\%-70\% FC ${ }_{\text {máx }}$, apoiando a saúde ativa. 0 caráter social e emocional das aulas de natação é, para a população adulta, muito positivo.

\section{REFERÊNCIAS}

1. Macejkova Y, et al. Didaktika plavania. Vysokoskolská ucebnica. Bratislava: ICM Agency; 2005.

2. Masaryková D. Diagnostic of training state by using standard swimming tests, blood tests and sporttesters. In: Scientific fundaments of human movement and sport practicy; 2005 Sep 16-18; Rimini, Itália. Bologna: Centro Universitario Sportivo Bolognese; 2005.

3. Ondrusova $L$, Macejkova $Y$. The influence of aqua-fitness elements on the aerobic skills of university students. In: Starosta W, O sinski W. New ideas in sport sciences - Curent issues and perspectives, part 2. Leszno: Wydawnictwo panstwowej Wyszej szkoly zawodowej; 2003.

4. Ondrusova L, Macejkova Y. Bencurikova L. The structure of motion activities and thei efficiency. In: Scientific fundaments of human movement and sport practicy; 2005 Sep 16-18; Rimini, Itália. Bologna: Centro Universitario Sportivo Bolognese; 2005.

5. Butts NK, Tucker M, et al. Physiologic responses to maximal treadmill and deep water running in men and women. Am J Sports Med. 1991; 62:236-9. 